\title{
Sensing Performance of Dielectric Sensor in Mixed Melting Polymer Pellets for \\ Waste Plastic Separation
}

\author{
Yusuke HIROSE, Chiba University, 1-33, Yayoi-cho, Inage-ku, Chiba-shi, Chiba 263-8522 \\ yuusuke.hirose@chiba-u.jp \\ Kazuaki HATA, Chiba University \\ Michiko SUGAWARA, Chiba University \\ Masahiro TAKEI, Chiba University
}

Keywords: Polymer, Impedance measurement, Dielectric properties, Waste plastic separation, Temperature dependence

\begin{abstract}
Physical value of dielectric properties among relative permittivity, dielectric loss and loss tangent of mixed melting polymer pellets were measured under temperature variance condition and measurement frequency from $0.5 \mathrm{MHz}$ to $5 \mathrm{MHz}$ in order to develop a realtime non-intrusive measurement technique for high performance waste plastic separation. Various volume fractions of Non-polar polyethylene (PE) and polar polyethylene terephthalate (PET) were used as the mixed polymer pellets. As the results, with the volume fractions of PET from 0.05 to 0.22 , as the temperature is increased up to melting point temperature and above mold temperature, the relative permittivity is decreased as the temperature is increased. However over melting temperature up to the mold temperature, the relative permittivity is slightly increased as the temperature is increased. On the other hand, the dielectric loss and loss tangent are not affected by the temperature variance, and the dielectric loss and loss tangent are increased as the measurement frequency is increased. As the conclusions, at molding temperature $180{ }^{\circ} \mathrm{C}(453 \mathrm{~K})$, the highest sensitivity of dielectric property is the relative permittivity. Meanwhile, the highest linearity relationship between PET volume fraction and predicted relative permittivity is achieved at the measurement frequency of $5 \mathrm{MHz}$. From relative permittivity measurement in these conditions, the performance to distinguish PET volume fraction 0.05 from PE volume fraction 0.95 is achieved in the mixed melting polymer pellets. Therefore, the relative permittivity measurement for waste plastic separation has a possibility to achieve the high purity recycled plastics as compared with conventional methods.
\end{abstract}




\section{Nomenclature}

$c$

$C_{0}$

D

$f$

H

$k_{B}$

$M$

n

$N_{A}$

$P$

$R$

$T$

$\tan \delta$

W

Greek letters

$\alpha$

$\varepsilon$

$\varepsilon^{\prime \prime}$

$\phi$

$\kappa$

$\mu$

$\rho$

\section{Subscript}

0

-deff

a

$c$

e

E

$g$

i

l

m

$\max$

$\min$

mold

$o$

p
Capacitance

F

Cell constant

F

Distance of experimental setup

$\mathrm{m}$

Measurement frequency

$\mathrm{Hz}$

Height of experimental setup

Boltzmann constant

$\mathrm{m}$

Molar mass

$\mathrm{J} / \mathrm{K}$

Data number

Avogadro constant

$\mathrm{kg} / \mathrm{mol}$

$1 / \mathrm{mol}$

Pressure

$\mathrm{Pa}$

Radius of cylindrical electric furnace

Temperature

Loss tangent

Width of experimental setup

K

$-$

$\mathrm{m}$

$\mathrm{C}^{2} \mathrm{~m}^{2} / \mathrm{J}$

$\mathrm{F} / \mathrm{m}$

Permittivity

Dielectric loss

Volume fraction

Conductivity

$\mathrm{S} / \mathrm{m}$

Permanent dipole moment

$\mathrm{Cm}$

Density of plastic

$\mathrm{kg} / \mathrm{m}^{3}$

Vacuum

Difference value

Air

Cylindrical electric furnace

Electric

Electrode

Glass cell

$i$-th data number

Low value

Melt value

Maximum value

Minimum value

Mold value

Atmosphere

Orientational value 


$\begin{array}{ll}P E & \text { Polyethylene value } \\ P E T & \text { Polyethylene terephthalate value } \\ r & \text { Relative value } \\ s & \text { Stray value } \\ v & \text { Vacuum container } \\ & \\ \text { Superscript } & \\ \text { predicted } & \text { Value from linear approximation } \\ \text { real } & \text { Value from experimental } \\ - & \text { Average }\end{array}$

\section{Introduction}

Recycling waste plastics are highly paid attention for sustainable society. Waste plastics from home and office include basically $90 \%$ major plastics such as polyethylene (PE), polystyrene (PS) and polypropylene (PP), and $10 \%$ minor plastics such as polyethylene terephthalate (PET) and polyvinyl chloride (PVC). A separation method from the mixed plastics is highly required to produce highly pure recycle plastic pellets. Conventional method is composed of two steps, which are a combination of an infrared separation method with air jet based on the refractive index difference value as first method, and a flotation separation method based on specific gravity difference value as second method. However, in the conventional method, the purity of recycled plastic pellets is usually less than $80 \%$. Therefore, the operation by workers hands is needed to increase the purity. Especially, the separated PE includes anyhow different types of plastics such as less than around $10 \%$ PET because of the separation performance limitation, resulting from the small difference in the refractive index and specific gravity between the mixtures. In order to achieve the high purity, third combination of separation method has been considered with integrating the in-line sensing of the mixture volume fraction as an extrusion process to produce recycle plastic pellets. The third combination separation method is needed a sensing technique to distinguish PET volume fraction in several kinds of mixed melting polymer pellets. However, the development is not already well established yet.

Generally, regarding in-line sensing for one type pure and virgin polymer, a dielectric sensor is often used to measure PE volume fraction of a granule flow by distinguish the relative permittivity distribution [1]. However, the dielectric sensor is strongly depended on temperature and measurement frequency. The characteristics of polymer dielectric properties such as relative permittivity, dielectric loss and loss tangent have been reported under molding temperature by a numerous researchers. Švorčík et al. obtained the relationship between relative permittivity and temperature for polymethyl methacrylate (PMMA) in composites to conclude that the relative permittivity is increased with temperature increase [2]. Neagu et al. measured a relative permittivity, dielectric loss and loss tangent with temperature and measurement frequency change for Nylon11; they concluded that the dielectric properties are increased 
with temperature increase, and they are decreased with measurement frequency increase [3]. Coburn et al. obtained the relative permittivity and dielectric loss against temperature and measurement frequency for Polyethylene terephthalate (PET). The change of relative permittivity and dielectric loss have two peaks from $73 \mathrm{~K}\left(-200^{\circ} \mathrm{C}\right)$ to $413 \mathrm{~K}\left(140^{\circ} \mathrm{C}\right)$. Also, the peak positions change due to the measurement frequency change [4]. In other words, the changes in dielectric properties depend on the polymer type, temperature and measurement frequency. However, the target temperatures of those dielectric properties from the scientific articles are extremely lower than melting temperature in an extruder for waste plastic separation. Therefore, from the engineering viewpoint, the dielectric properties of mixed melting polymer pellets are highly demanded to attain a high purity.

In this study, the optimal dielectric properties (i.e. relative permittivity, dielectric loss and loss tangent) of melting polymer pellets with various PET volume fractions are determined in order to indicate the detailed dielectric properties above melting temperature. Moreover, the optimal measurement frequency is determined under various conditions of temperature and measurement frequency in

order to develop a best performance sensing technique to distinguish PET volume fraction in the mixture of non-polar polyethylene (PE) and polar polyethylene terephthalate (PET) for waste plastic separation.

\section{Proposed sensing technique of mixed melting polymer for waste plastic separation}

Figure 1 shows a proposed sensing technique of mixed melting polymer for waste plastic separation, which consists of a pulverizer, an infrared separation machine, a flotation separation machine, a drier, a deforming extruder, a sensing extruder, a dielectric sensor, a RC meter, a granulator, and a remover. The dielectric sensor is attached in a periphery of discharge pipe of the sensing extruder. At first, the recovered waste plastics are pulverized by a pulverizer. The pulverized waste plastics are separated by infrared separation method and flotation separation method. After the separations, the waste plastics are dried by a drier. The dried waste plastics are melted and fluidized in the deforming and the sensing extruders at molding temperature $T_{\text {mold }}[\mathrm{K}]$. In the sensing extruder, the sensor measures PET volume fraction $\phi_{P E T}[-]$ by physical value of dielectric properties, the low purity parts of melt polymer are removed by a remover with air jet after granulation. This third separation technique is assumed to contribute for producing highly pure recycled pellets. In this technique, three dielectric properties are selected, which are relative permittivity $\varepsilon_{r}[-]$, dielectric loss $\varepsilon^{\prime \prime}[-]$ and loss $\operatorname{tangent} \tan \delta[-]$. The relationship between $\varepsilon_{r}[-]$ and temperature $T[\mathrm{~K}]$ in a polar polymer such as PET are expressed by Debye equation,

$\frac{\varepsilon_{r}-1}{\varepsilon_{r}+2}=\frac{\rho N_{A}}{3 \varepsilon_{0} M}\left(\alpha_{e}+\alpha_{p}\right)=\frac{\rho N_{A}}{3 \varepsilon_{0} M}\left(\alpha_{e}+\frac{\mu^{2}}{3 k_{B} T}\right)$ 
where $\varepsilon_{0}$ is vacuum permittivity $[\mathrm{F} / \mathrm{m}], M$ is molar mass $[\mathrm{kg} / \mathrm{mol}], \rho$ is density $\left[\mathrm{kg} / \mathrm{m}^{3}\right], N_{A}$ is Avogadro constant $=6.02 \times 10^{23} 1 / \mathrm{mol}, k_{B}$ is Boltzmann constant $=1.38 \times 10^{-23} \mathrm{~J} / \mathrm{K}, \mu$ is permanent dipole moment $[\mathrm{Cm}], \alpha_{e}$ is electric polarizability $\left[\mathrm{C}^{2} \mathrm{~m}^{2} / \mathrm{J}\right], \alpha_{p}$ is orientational polarizability $\left[\mathrm{C}^{2} \mathrm{~m}^{2} / \mathrm{J}\right]$. The trend of Debye equation indicates that $\varepsilon_{r}$ is decreased as $T$ is increased under $\rho$ is constant [5]. In the case of non-polar polymer such as PE, $\alpha_{p}$ term is deleted because $\mu$ is zero; namely, Clausius-Mosotti equation is expressed by

$\frac{\varepsilon_{r}-1}{\varepsilon_{r}+2}=\frac{\rho}{M} \frac{N_{A}}{3 \varepsilon_{0}} \alpha_{e}$

From this equation, $\varepsilon_{r}$ is decreased as $\rho$ is decreased [6].

Meanwhile, $\varepsilon^{\prime \prime}$ is expressed by

$\varepsilon^{\prime \prime}=\frac{\kappa}{2 \pi f \varepsilon_{0}}$

$\kappa$ is conductivity $[\mathrm{S} / \mathrm{m}]$ which depends on $T$, and $f$ is measurement frequency $[\mathrm{Hz}]$. From this equation, $\varepsilon^{\prime \prime}$ depends on $T$ and $f$.

$\tan \delta$ is the ratio of $\varepsilon_{r}$ and $\varepsilon^{\prime \prime}$ as [5]. And also depend on $T$ and $f$;

$\tan \delta=\frac{\varepsilon^{\prime \prime}}{\varepsilon_{r}}$

Fig. 1 The Proposed Sensing technique of mixed melting polymers

\section{Experiments}

\subsection{Experimental setup}

Figure 2 (a) shows the overall schematic diagram of experimental setup which was composed of an electric furnace (Model MCK111010, Motoyama Co.Ltd Japan), a petri dish, a multi controller (Model FKC-12, TGK Japan), two glass cells, an impedance analyzer (Model IM3570, Hioki E.E. Corporation Japan), a four-terminal probe (L2000, Hioki E.E. Corporation Japan), three thermocouples (Model KTO-16200 type K, As One Corporation Japan), a data logger/switching unit (Model 34970A, Agilent technologies company USA) and a vacuum container. The atmosphere in cylindrical electric furnace with the radius $R=50 \mathrm{~mm}$ and the height $H_{c}=150 \mathrm{~mm}$ was heated up homogeneously by heating wires located in the side wall. The petri dish was located at top of the electric furnace for temperature stabilization. The multi controller was regulated the inside temperature for each $1.00 \mathrm{~K}\left(1.00{ }^{\circ} \mathrm{C}\right)$ by means of proportional integral derivative control. Figure 2 (b) shows the grass cells, electrodes, K-type thermocouples, coaxial cables, and 
crocodile clips inside the electric furnace. Both glass cells which were a capacitance measurement cell and a temperature measurement cell were made of a quartz glass with $D_{g} \times H_{g} \times W=10.0 \mathrm{~mm} \times 50.0 \mathrm{~mm} \times 50.0 \mathrm{~mm}$, and had two copper electrodes with $H_{E} \times W=$ $10.0 \mathrm{~mm} \times 50.0 \mathrm{~mm}$ inside the larger wall. The impedance analyzer measured the impedance and phase angle with various measurement frequencies $f$. The end of four terminals probe from the impedance analyzer was clipped by using crocodile clips at the top of the copper electrodes in the glass cell. One thermocouple was located in the other glass cell, and the other thermocouple was located at the atmosphere. Both thermocouples were connected to the data logger/switching unit to record the temperature $T$. The vacuum container consists of a pressure gauge, a vacuum valve, a leak valve, a vacuum pump and a brick. The vacuum container with the height $H_{v}=545 \mathrm{~mm}$, the width $W_{v}=390 \mathrm{~mm}$, the internal depth $D_{v}=446 \mathrm{~mm}$ was used for sample preparation as explained in 3.2 section in order to avoid bubble inclusions during the measurement. The vacuum valve was connected to a vacuum pump and the leak valve was used for atmospheric release. The furnace was located on the bricks for thermal insulation.

(a) Overall schematic diagram

(b) Inside electric furnace

Fig. 2 Experimental setup

\subsection{Sample Preparation Procedure}

Figure 3 shows the polyethylene (PE) and polyethylene terephthalate (PET) pellets, which are elliptic cylinder shape. Table 1 shows two kinds of polymer pellet properties which are non-polar PE (LJ902, Japan polyethylene Corporation) and polar PET (TRN8380AC, Teijin limited Japan). Polar plastics have permanent dipole moment $\mu$, which is represent the deviation of positive and negative charge of the molecule in the absence of an electric field. Based on this fact, permittivity of polar plastics is larger than nonpolar plastics [7]. Also, the molding temperature $T_{m o l d}$ must be more than the melting temperature $T_{m}$ and less than the deterioration temperature. The pellet size indicates the value of the longest diameter, the shortest diameter, and height. The \pm value in parenthesis is deviation. Table 2 shows four samples with various combination of each PET volume fraction $\phi_{P E T}$ around $10 \%$ from the viewpoints of real separation situation. As a reference, Sample 4 is only PET, which has the maximum $\phi_{P E T}=1.00$. 
At first in the procedure, the pellets as shown in Fig. 3 were put into both glass cells in the electric furnace. The furnace was covered with the petri dish, and the vacuum container was closed. The vacuum pump was operated from atmospheric pressure $P_{0}=0.00 \mathrm{MPa}$ Gauge to low pressure $P_{l}=-0.09 \mathrm{MPa}$ Gauge. The maximum temperature inside the furnace was set as $473 \mathrm{~K}\left(200{ }^{\circ} \mathrm{C}\right)$ for Samples 1 to 3 and $523 \mathrm{~K}\left(250^{\circ} \mathrm{C}\right)$ for Sample 4 to be held for 40 minutes to achieve a constant temperature for each sample. According to the thermal diffusion differential equation, the 40 minutes are confirmed to be steady and uniform temperature condition. After 40 minutes of holding, the vacuum valve was closed and the leak valve was opened. The pressure inside the vacuum container was naturally reached to $P_{0}$. After reaching $P_{0}$, the furnace and the pump were turned off. Thus, the temperature was decreased down to room temperature; the mixed samples without any bubbles were obtained. All sample volumes were $6,000 \mathrm{~mm}^{3}$.

Fig. 3 PE and PET pellets

Table 1 Polymer properties of and experiment condition [7] [8]

\begin{tabular}{ccc}
\hline & PE & PET \\
\hline Chemical formula & $-\left(\mathrm{C}_{2} \mathrm{H}_{4}\right)_{\mathrm{n}^{-}}$ & $-\left(\mathrm{C}_{10} \mathrm{H}_{10} \mathrm{O}_{4}\right)_{\mathrm{n}^{-}}$ \\
\hline Polarity & Non-polarity & Polarity \\
\hline Crystalline/Amorphous & Crystalline & Crystalline \\
\hline Melting temperature $T_{m}[\mathrm{~K}]$ & 375 & 498 \\
\hline Molding temperature $T_{\text {mold }}[\mathrm{K}]$ & 453 & 543 \\
\hline & $3.98( \pm 0.0908) \times 2.67$ & $2.98( \pm 0.102) \times 2.59( \pm 0.228) \times$ \\
Pellet size $( \pm$ deviation $)$ & $( \pm 0.110) \times 3.11( \pm 0.169) \mathrm{mm}$ & $3.08( \pm 0.279) \mathrm{mm}$ \\
\hline
\end{tabular}

Table 2 PET volume fraction of 4 samples

\begin{tabular}{ccc}
\hline & PET volume fraction of PE 1- $\phi_{P E T}[-]$ & PET volume fraction $\phi_{P E T}[-]$ \\
\hline Sample 1 & 0.95 & 0.05 \\
\hline Sample 2 & 0.90 & 0.10 \\
\hline Sample 3 & 0.78 & 0.22 \\
\hline
\end{tabular}




$\begin{array}{lll}\text { Sample } 4 & 0.00 & 1.00\end{array}$

\subsection{Experimental Conditions and Methods}

The experimental maximum temperature $T_{\max }$ were $473 \mathrm{~K}\left(200{ }^{\circ} \mathrm{C}\right)$ for Samples 1 to 3 and $523 \mathrm{~K}\left(250{ }^{\circ} \mathrm{C}\right)$ for Sample 4 after the sample preparation procedure. The polymer $T_{\max }$ was set above PE molding temperature $T_{m o l d-P E}=453 \mathrm{~K}\left(180{ }^{\circ} \mathrm{C}\right)$ for PE, and then the temperature was held for 40 minutes in $T_{\max }$. After that, the electric furnace was turned off to start the measurements during the natural cooling until approximate $T_{\min }=253 \mathrm{~K}\left(30^{\circ} \mathrm{C}\right)$. During the natural cooling, the impedance analyzer measured the capacitance $c$ and loss tangent $\tan \delta$ with various measurement frequencies $f$ from $0.5 \mathrm{MHz}$ to $5 \mathrm{MHz}$ after open/short compensation. The measurement values were averaged by each $10 \mathrm{~K}\left(10^{\circ} \mathrm{C}\right)$ decrement. Calibration of $c$ is necessary because the stray capacitance $c_{s}$ occurs from the electrodes shapes and the cables. $c_{s}$ can be described by

$c_{s}=c_{a}-\varepsilon_{a} C_{0}$

where $c_{a}$ is the air capacitance and $C_{0}$ is the cell constant [9], and $\varepsilon_{a}$ is the air relative permittivity, which is equal to 1.00. Meanwhile, $C_{0}$ is described by

$C_{0}=\varepsilon_{0} \frac{H_{E} \times W}{D_{g}}$

where $H_{E} \times W$ is the electrode area and $D_{g}$ is the distance between electrodes. In order to remove $c_{s}$, the polymers relative permittivity $\varepsilon_{r}$ is calibrated by

$\varepsilon_{r}=\frac{c-c_{s}}{C_{0}}$

This calibration is performed in each temperature $T$ because $c_{s}$ is dependent on each $T$. The data logger/switching unit measured $T$ synchronizedly at the same time as the impedance analyzer measurement.

\section{Experimental results}

\subsection{Relative Permittivity in Mixed PE and PET}

Figures 4 (a) and (b) show the relationship between temperature $T$ and relative permittivity $\varepsilon_{r}$ as a parameter of measurement frequency $f=0.5 \mathrm{MHz}, 1 \mathrm{MHz}, 2 \mathrm{MHz}, 3 \mathrm{MHz}, 4 \mathrm{MHz}$ and $5 \mathrm{MHz}$ in the case of Sample $1\left(\phi_{P E T}=0.05\right)$, Sample $2\left(\phi_{P E T}=0.10\right)$, Sample $3\left(\phi_{P E T}=0.22\right)$, and Sample $4\left(\phi_{P E T}=1.00\right)$, respectively. From the results of Sample 1 to 3, basically, $\varepsilon_{r}$ is dependent on $T ; \varepsilon_{r}$ is decreased as $T$ is increased up to around PE melting temperature $T_{m-P E}$ because the polymer volume is expanded, the polymer 
density $\rho$ is decreased based on Eq.(2). Above $T_{m-P E}$ up to PE mold temperature $T_{m o l d-P E}, \varepsilon_{r}$ is slightly increased as $T$ is increased. Above $T_{\text {mold-PE }}, \varepsilon_{r}$ is slightly decreased as $T$ is increased. In the case of Sample $4\left(\phi_{P E T}=1.00\right), \varepsilon_{r}$ curve indicates a peak at $T=423 \mathrm{~K}$ $\left(150^{\circ} \mathrm{C}\right)$. In polar polymer, the effect of orientation polarization $\alpha_{p}$ is dominant, and several relaxations are shown in wide temperature region. The corresponding temperature of each relaxation is almost constant irrespective of $f$. Namely, the temperature dependence of $\varepsilon_{r}$ in PET is more complex than that in PE. Table 3 shows $\varepsilon_{r}$ gradient of Samples 1 to 3 up to $T_{m-P E}$. According to Table 3, the gradient is decreased as $\phi_{P E T}$ is increased. On the other hand, in the polar polymer, the permanent dipole moment $\mu$ represents the deviation of positive and negative charge of the molecules constituting the polymer without an electric field. A large number of dipole lower temperature zone can easily orient in the external electric field direction because $\varepsilon_{r}$ at $T$ is increased. That proceeds polarization because dipoles is easy to move, $\varepsilon_{r}$ tends to increase with $T$ increase. However, $\varepsilon_{r}$ is tend to decrease with increasing $T$ over the orderdisorder transition temperature which is one of the phase transition temperature. This is caused by a thermal disturbance of the dipole orientation and further density decrease [5].

$$
\text { ( } \alpha) \phi_{P E T}=0.05 \text { and } \phi_{P E T}=0.10
$$

(b) $\phi_{P E T}=0.22$ and $\phi_{P E T}=1.00$

Fig. 4 Temperature dependence of relative permittivity $\varepsilon_{r}$

Table 3 Line gradient below $T_{m-P E}$ in $f=5 \mathrm{MHz}$

\begin{tabular}{cc}
\hline & gradient $\times 10^{-3}[1 / \mathrm{K}]$ \\
\hline Sample 1 & -3.64 \\
\hline Sample 2 & -2.89 \\
\hline Sample 3 & -1.50 \\
\hline
\end{tabular}

\subsection{Dielectric Loss in Mixed PE and PET}

Figures 5 (a) and (b) show the relationship between temperature $T$ and dielectric loss $\varepsilon^{\prime \prime}$ as a parameter of measurement frequency $f=$ $0.5 \mathrm{MHz}, 1 \mathrm{MHz}, 2 \mathrm{MHz}, 3 \mathrm{MHz}, 4 \mathrm{MHz}$ and $5 \mathrm{MHz}$ in the case of Sample 1 (PET volume fraction $\left.\phi_{P E T}=0.05\right)$, Sample $2\left(\phi_{P E T}=\right.$ 
$0.10)$, Sample $3\left(\phi_{P E T}=0.22\right)$, and Sample $4\left(\phi_{P E T}=1.00\right)$, respectively. From mixed sample (Sample 1 to 3$)$ results, $\varepsilon^{\prime \prime}$ are almost constant regardless of $T$. As $\phi_{P E T}$ and $f$ are increased, the variation and value of $\varepsilon^{\prime \prime}$ become larger. In the case of Sample 4, the peak value of $\varepsilon^{\prime \prime}$ to $T$ is shown as a dot-and-dash line, and the peak potion tends to move to higher temperature as $f$ is increased. The peaks exhibit the correspondence to primary relaxation. In terms of the polar polymers, $\varepsilon^{\prime \prime}$ shows a graph of a mountain-shaped having the maximum value at the position where relative permittivity $\varepsilon_{r}$ peaks at the order-disorder transition temperature. On the other hand, in the non-polar polymer indicates only ionic polarization and electronic polarization. For this reason, $\varepsilon^{\prime \prime}$ change of Samples 1 to 3 is smaller than the Sample 4 change.

$$
\text { (a) } \phi_{P E T}=0.05 \text { and } \phi_{P E T}=0.10
$$

(b) $\phi_{P E T}=0.22$ and $\phi_{P E T}=1.00$

Fig. 5 Temperature dependence of dielectric loss $\varepsilon^{\prime \prime}$

\subsection{Tangent Delta in Mixed PE and PET}

Figures 6 (a) and (b) show the relationship between temperature $T$ and loss tangent $\tan \delta$ as a parameter of measurement frequency $f=$ $0.5 \mathrm{MHz}, 1 \mathrm{MHz}, 2 \mathrm{MHz}, 3 \mathrm{MHz}, 4 \mathrm{MHz}$ and $5 \mathrm{MHz}$ in the case of Sample 1 (PET volume fraction $\left.\phi_{P E T}=0.05\right)$, Sample $2\left(\phi_{P E T}=\right.$ $0.10)$ and Sample $3\left(\phi_{P E T}=0.22\right)$, and Sample $4\left(\phi_{P E T}=1.00\right)$, respectively. From Sample 1 to Sample 3 results, tan $\delta$ is almost constant irrespective of $T$. As $\phi_{P E T}$ and $f$ are increased, the variation and value of $\tan \delta$ become larger. In the case of polar plastics (Sample 4), the peak value of $\tan \delta$ in terms of $T$ is shown as a dot-and-dash line, and the peak potion tends to move to higher temperature as $f$ is increased. The peaks exhibit the correspondence to primary relaxation. Also, the amount of $\tan \delta$ peak movement against $T$ is smaller than the amount of $\varepsilon^{\prime \prime}$.

( $\alpha) \phi_{P E T}=0.05$ and $\phi_{P E T}=0.10$

(b) $\phi_{P E T}=0.22$ and $\phi_{P E T}=1.00$ 
Fig. 6 Temperature dependence of loss tangent $\tan \delta$

\section{Discussion}

\subsection{Optimal dielectric properties in mixed melting polymer pellets}

The optimal physical value of dielectric properties among relative permittivity $\varepsilon_{r}$, dielectric loss $\varepsilon^{\prime \prime}$ and loss $\operatorname{tangent} \tan \delta$ should be selected in mixed melting polymer pellets to achieve the best performance for waste plastic separation from the viewpoint of high sensitivity and linearity. Figure 7 and 8 show the relationship between PET volume fraction $\phi_{P E T}$ and $\varepsilon_{r}$ at PE molding temperature $T_{\text {mold }-P E}=453 \mathrm{~K}\left(180^{\circ} \mathrm{C}\right)$, the relationship between $\phi_{P E T}$ and $\varepsilon^{\prime \prime}$ at $T_{\text {mold-PE}}$, and the relationship between $\phi_{P E T}$ and $\tan \delta$ at $T_{m o l d-P E}$. From these figures, $\varepsilon_{r}$ is linearly increased as $\phi_{P E T}$ is increased; however, $\varepsilon^{\prime \prime}$ and $\tan \delta$ are not linearly increased. Concretely, $\varepsilon^{\prime \prime}$ and $\tan \delta$ is increased rapidly from $\phi_{P E T}=0.10$ to $\phi_{P E T}=0.22$. Also, different values between the dielectric properties at $\phi_{P E T}=0.05$ and the dielectric properties at $\phi_{P E T}=0.1$ must be required in this proposed method because the conventional separation method cannot achieve less than $10 \%$ PET sensing. Figure 9 shows the relationship between the difference values of $\left|\varepsilon_{r-d i f f}\right|, \mid \varepsilon^{\prime \prime}$-diff $\mid$ and $\left|\tan \delta_{\text {-diff }}\right|$ versus $f$ at $T_{\text {mold-PE. }}\left|\varepsilon_{r-d i f f}\right|$ indicates the different value between $\varepsilon_{r}\left(\phi_{P E T}=0.10\right)$ and $\varepsilon_{r}\left(\phi_{P E T}=0.05\right)$. The $\mid \varepsilon^{\prime \prime}$-diff $\mid$ indicates the different value between $\varepsilon^{\prime \prime}\left(\phi_{P E T}=0.10\right)$ and $\varepsilon^{\prime \prime}\left(\phi_{P E T}=0.05\right)$. The $\left|\tan \delta_{\text {-diff }}\right|$ indicates the different value between $\tan \delta\left(\phi_{P E T}=0.10\right)$ and $\tan \delta\left(\phi_{P E T}=\right.$ 0.05). Among the difference values, $\left|\varepsilon_{r-\text {-diff }}\right|$ is the largest among $\left|\varepsilon_{r-\text {-diff }}\right|, \mid \varepsilon^{\prime \prime}$-diff $\mid$ and $\left|\tan \delta_{\text {-diff }}\right|$ regardless of $f$. From the viewpoint of high sensitivity, $\varepsilon_{r}$ is most appropriately chosen among three dielectric properties in mixed melting polymer pellets in order to achieve the best performance.

Table 4 shows the linear approximation with a slope $A$ and an intercept $B$ between $\phi_{P E T}$ and $\varepsilon_{r}$ as a parameter of $f$. From the viewpoint of high linearity, $f=5 \mathrm{MHz}$ is the optimal condition because of the largest gradient of the linear approximation.

Fig. 7 Relative permittivity $\varepsilon_{r}$ and dielectric loss $\varepsilon^{\prime \prime}$ versus $\phi_{P E T}$ in various $f$ at $T_{\text {mold-PE }}$

Fig. 8 Relative permittivity $\varepsilon_{r}$ and loss tangent $\tan \delta$ versus $\phi_{P E T}$ in various $f$ 
Fig. 9 Difference of dielectric properties $\left|\varepsilon_{r-\text {-diff }}\right|, \mid \varepsilon^{\prime \prime}$-diff $\mid$ and $\left|\tan \delta_{\text {-diff }}\right|$ between $\phi_{P E T}=0.1$ and $\phi_{P E T}=0.05$ against $f$

Table 4 Liner approximation between $\phi_{P E T}$ and $\varepsilon_{r}$

\begin{tabular}{ccc}
\hline$T_{\text {mold } P \text { PE }}=453 \mathrm{~K}\left(180{ }^{\circ} \mathrm{C}\right)$ & $A$ & $B$ \\
\hline $0.5 \mathrm{MHz}$ & 3.39 & 1.91 \\
\hline $1 \mathrm{MHz}$ & 3.39 & 1.92 \\
\hline $2 \mathrm{MHz}$ & 3.39 & 1.93 \\
\hline $3 \mathrm{MHz}$ & 3.41 & 1.98 \\
\hline $4 \mathrm{MHz}$ & 3.43 & 2.03 \\
\hline $5 \mathrm{MHz}$ & 3.48 & 2.08 \\
\hline
\end{tabular}

\subsection{Sensing performance in mixed melting polymer pellets}

According to the discussion of 5.1 section, in terms of the high sensitivity and the linearity, the relative permittivity $\varepsilon_{r}$ with measurement frequency $f=5 \mathrm{MHz}$ is the best physical value among dielectric properties to detect PET volume fraction $\phi_{P E T}$ in waste plastic separation process. However, the best physical value is necessary to examine the measurement standard deviation $\sigma$ from the atmosphere and the measurement system and the difference between real $\phi_{P E T}$ and linear approximation in Table 4 . Also, the optimal $f$ condition is discussed at the fixed PE molding temperature $T_{\text {mold } P E}=453 \mathrm{~K}\left(180{ }^{\circ} \mathrm{C}\right)$ to recognize how much minimum $\phi_{P E T}$ is in mixed melting polymer pellets. First, $\sigma$ of $\varepsilon_{r}$ at each $f$ and various $\phi_{P E T}$ at the fixed room temperature is

$\sigma=\sqrt{\frac{1}{n} \sum_{i=1}^{i=n}\left(\overline{\varepsilon_{r}}-\varepsilon_{r i}\right)^{2}}$

where $n$ is the data number, $\varepsilon_{r i}$ is the relative permittivity of $i$-th data number, and $\overline{\varepsilon_{r}}$ is the 100 times average of measured $\varepsilon_{r}$. Figure 10 shows the relationship between $f$ and $\sigma$ in various $\phi_{P E T}$. From this figure, $f=0.5 \mathrm{MHz}$ has the smallest $\sigma$ value.

Furthermore, the accuracy order of predicted PET volume fraction $\phi_{P E T}^{\text {predicted }}$ is analyzed based on $\sigma . \phi_{P E T}^{\text {predicted }}$ is defined from the value which is calculated from the linear approximation as showed in Table 4 and the $i$-th measured $\varepsilon_{r i}$. In the case of normal probability distribution, when the $i$-th measured $\varepsilon_{r i}$ is under $95.45 \%$, the probability has a range of

$\varepsilon_{r}-2 \sigma \leq \varepsilon_{r i} \leq \varepsilon_{r}+2 \sigma$

Because of 
$\phi_{P E T}{ }^{\text {predicted }}=\frac{\varepsilon_{r i}-B}{A}$

$\phi_{P E T^{\text {predicted }}}$ has a range of

$\frac{\varepsilon_{r}-2 \sigma-B}{A} \leq \phi_{P E T}^{\text {predicted }} \leq \frac{\varepsilon_{r}+2 \sigma-B}{A}$

Therefore, the difference between $\phi_{P E T}^{\text {predicted }}$ and $\phi_{P E T}$ real

$\frac{\phi_{P E T}^{r \text { real }}-\frac{\varepsilon_{r}-2 \sigma-B}{A}}{\phi_{P E T}^{\text {real }}} \times 100 \leq \frac{\phi_{P E T}^{\text {real }}-\phi_{P E T}^{\text {predicted }}}{\phi_{P E T}^{\text {real }}} \times 100 \leq \frac{\phi_{P E T}^{r e a l}-\frac{\varepsilon_{r}+2 \sigma-B}{A}}{\phi_{P E T}^{\text {real }}} \times 100$

Figure 11 shows the relationship between $f$ and the difference value which is dependent on $f$. The optimal $f$ showing the smallest difference value is $f=5 \mathrm{MHz}$. The difference values in $f=5 \mathrm{MHz}$ in the case of $\phi_{P E T}{ }^{\text {real }}=0.05,0.1,0.22$ and 1.0 are $25.1,4.68,9.41$ and $0.346 \%$, respectively. Therefore, this study proposes a new high performance sensing technique of mixed melting polymer pellets to distinguish $\phi_{P E T}^{\text {real }}=0.05$ from the PE volume fraction 0.95 by using $\varepsilon_{r}$ with optimal $f=5 \mathrm{MHz}$.

Fig. 10 Standard deviation of relative permittivity against measurement frequency in various $\phi_{P E T}$

Fig. 11 Relationship between $f$ and difference value from $\phi_{P E T}$ real to $\phi_{P E T}$ predicted

\section{CONCLUSIONS}

Physical values of dielectric properties among relative permittivity $\varepsilon_{r}$, dielectric loss $\varepsilon^{\prime \prime}$ and loss tangent $\tan \delta$ in melting mixture of PE and PET were measured in various measurement frequency $f$ from $0.5 \mathrm{MHz}$ to $5 \mathrm{MHz}$ in order to indicate the dielectric properties above melting temperature and to develop a new high performance sensing technique for waste plastic separation. The conclusions are obtained as follows.

1) In PET volume fraction from $\phi_{P E T}=0.05$ to $\phi_{P E T}=0.22, \varepsilon_{r}$ is decreased as $T$ is increased up to around PE melting temperature $T_{m-P E}$.

Over $T_{m-P E}$ up to PE mold temperature $T_{m o l d-P E}, \varepsilon_{r}$ is slightly increased as $T$ is increased. Over $T_{m o l d-P E}, \varepsilon_{r}$ is slightly decreased as $T$ is increased. $\varepsilon^{\prime \prime}$ and $\tan \delta$ are almost constant irrespective of $T$. As $\phi_{P E T}$ and $f$ are increased, the variation and value of $\varepsilon^{\prime \prime}$ and $\tan \delta$ 
becomes larger. In the case of $\phi_{P E T}=1.00, \varepsilon_{r}$ curve indicates a peak at $T=423 \mathrm{~K}\left(150^{\circ} \mathrm{C}\right)$. The peak potions of $\varepsilon^{\prime \prime}$ and $\tan \delta$ move to higher temperature as $f$ is increased. Also, the movement amount of $\tan \delta$ peak against $T$ is smaller than the movement amount of $\varepsilon^{\prime \prime}$.

2) From the viewpoint of high sensitivity, $\varepsilon_{r}$ is the best physical value among the three dielectric properties in mixed melting polymer pellets to achieve the best performance. From the viewpoint of high linearity, $f=5 \mathrm{MHz}$ is the optimal condition because of the largest gradient of the linear approximation at the molding temperature $T_{\text {mold- } P E}=180^{\circ} \mathrm{C}$.

3) The difference values between real PET volume fraction $\phi_{P E T}{ }^{\text {real }}$ and predicted PET volume fraction $\phi_{P E T}$ predicted in $f=5 \mathrm{MHz}$ in the case of $\phi_{P E T}^{\text {real }}=0.05,0.1,0.22$ and 1.0 are $25.1,4.68,9.41$ and $0.346 \%$, respectively. This study proposes a new high performance sensing technique of mixed melting polymer pellets to distinguish $\phi_{P E T}$ real $=0.05$ from the PE volume fraction 0.95 by using $\varepsilon_{r}$ with optimal $f=5 \mathrm{MHz}$.

\section{ACKNOWLEDGMENTS}

This research was supported by Adaptable and Seamless Technology Transfer Program through Target-driven R\&D, Japan Science and Technology Agency (\#AS251Z00116K).

\section{REFFERENCES}

[1] T. Zhao and M. Takei, Design of Plug Formation Detector Based on Capacitance Measurement Technique, Advanced Powder Technology, Vol.19, No.6 (2008) pp.559-572

[2] V. Švorčík, J. Králová, , V. Rybka, J. Plešek, J. Červená, V. Hnatowicz, Temperature dependence of the permittivity of polymer composites, Journal of Polymer Science, Part B: Polymer Physics, 39 (2001) 831-834.

[3] R.M. Neagu, E. Neagu, N. Bonanos, P. Pissis, Electrical conductivity studies in nylon 11, Journal of Applied Physics, 88 (2000) 6669-6677.

[4] J. Coburn, R. Boyd, Dielectric relaxation in Poly(ethylene terephthalate), Macromolecules, 19(8) (1986) 2238-2245.

[5] H. Fröhlich, Theory of dielectrics, 2nd ed., Oxford University press, London, 1958.

[6] J.E. Mark, A. Eisenberg, W.W. Graessley, L. Mandelkern, E. T. Samulski, J.L. Koenig and G.D. Wignall, Physical properties of polymers, 2nd ed., United Book Press, Baltimore, 1993.

[7] J. Bicerano, Prediction of polymer properties, Marcel Dakker, Inc., New York, 1993.

[8] D.W. Van Krevelen, Properties of polymers, Elesevier Scientific Publishing Company, Amsterdam, 1976. 
[9] J. H. Park, C. S. Kim, B. C. Choi, K. Y. Ham, The correlation of the complex dielectric constant and blood glucose at low frequency, Biosensors and Bioelectronics, 19 (2003) 321-324. 


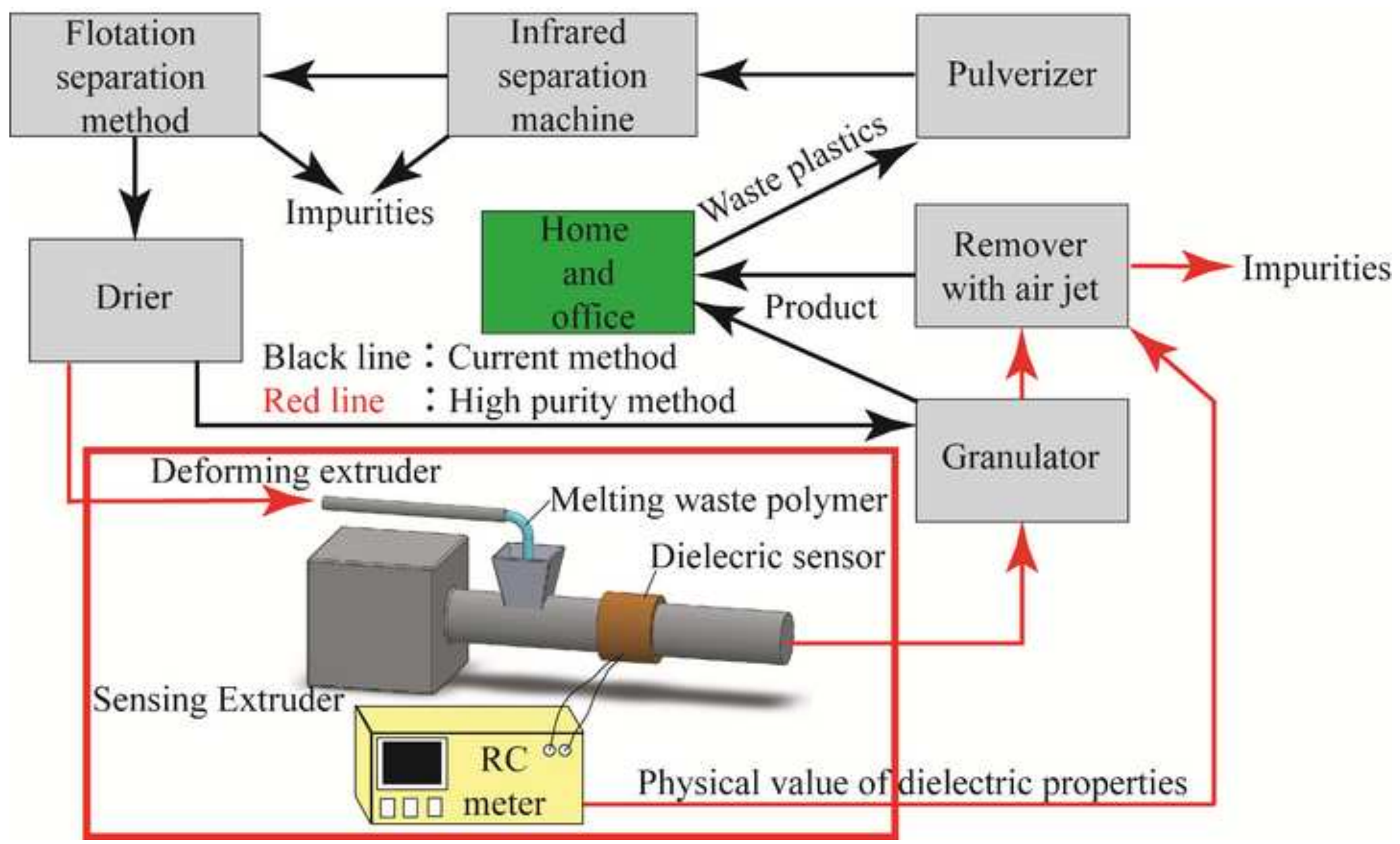




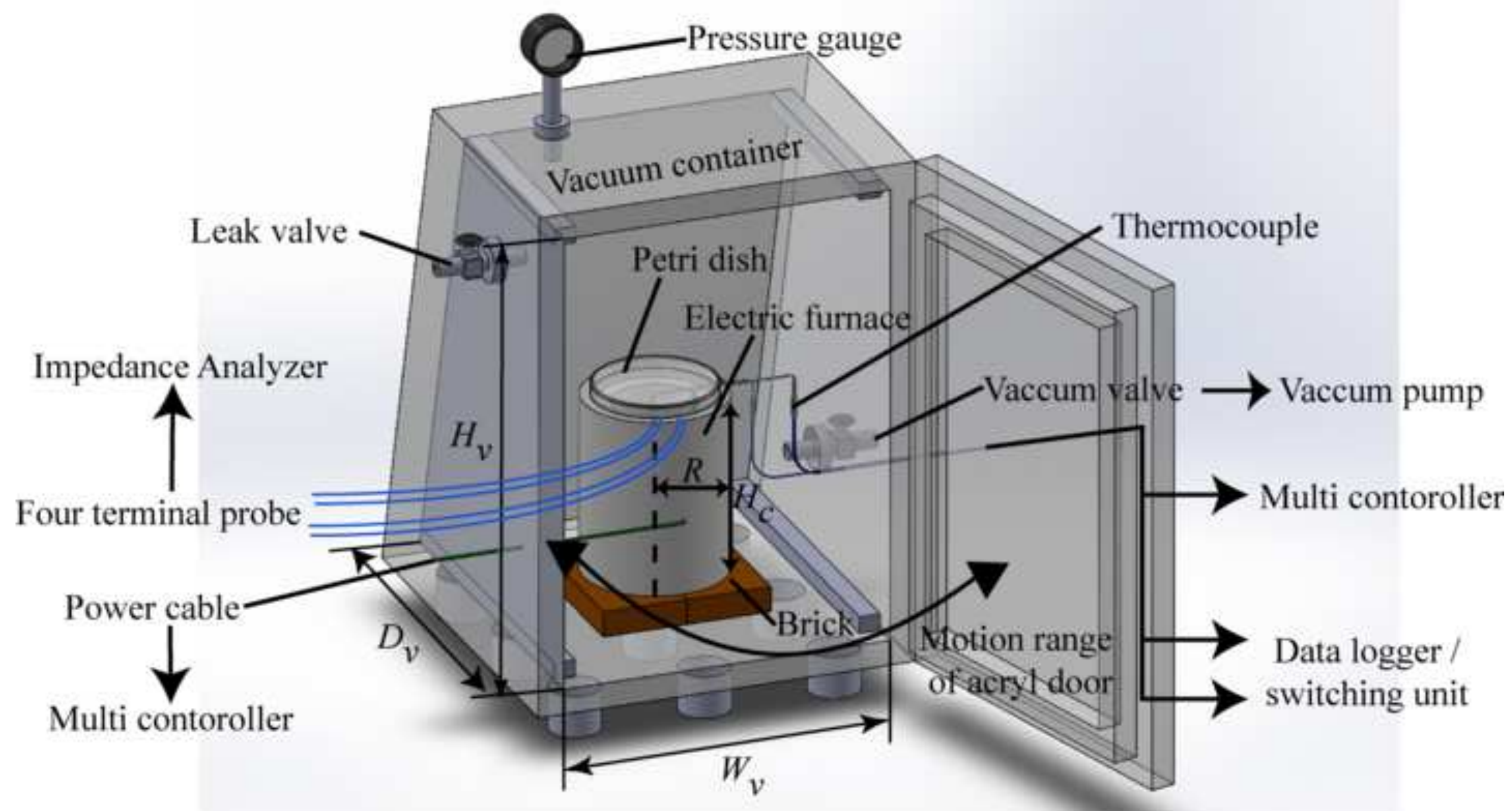




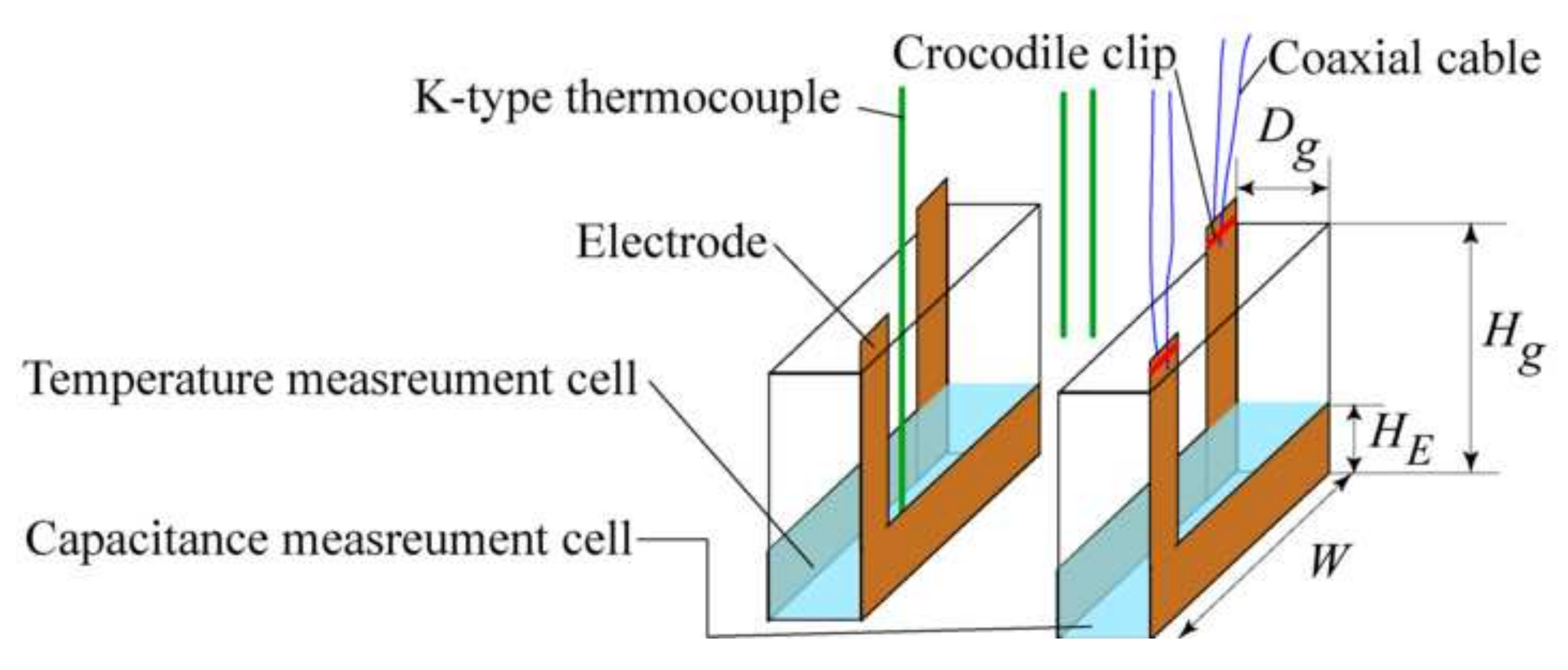

Temperature measreument cell

Capacitance measreument cell 


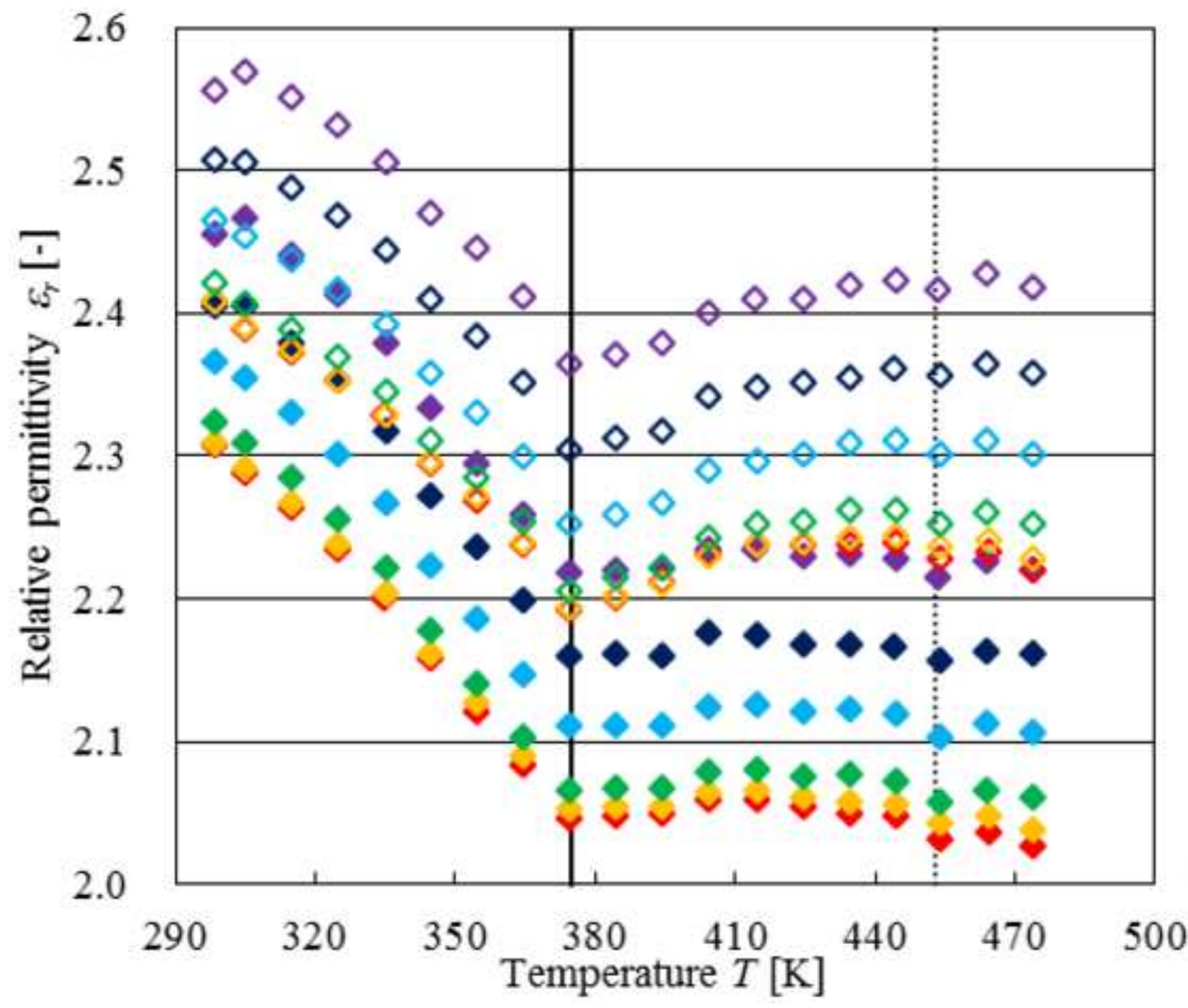

$\phi_{P E T}=0.05$

- $0.5 \mathrm{MHz}$

- $1 \mathrm{MHz}$

- $2 \mathrm{MHz}$

- $3 \mathrm{MHz}$

- $4 \mathrm{MHz}$

- $5 \mathrm{MHz}$

$\phi_{P E T}=0.10$

$\diamond \quad 0.5 \mathrm{MHz}$

$1 \mathrm{MHz}$

$2 \mathrm{MHz}$

$3 \mathrm{MHz}$

$\diamond \quad 4 \mathrm{MHz}$

$\diamond \quad 5 \mathrm{MHz}$

Temperature

$T_{m-P E}$

$T_{\text {mold-PE }}$ 


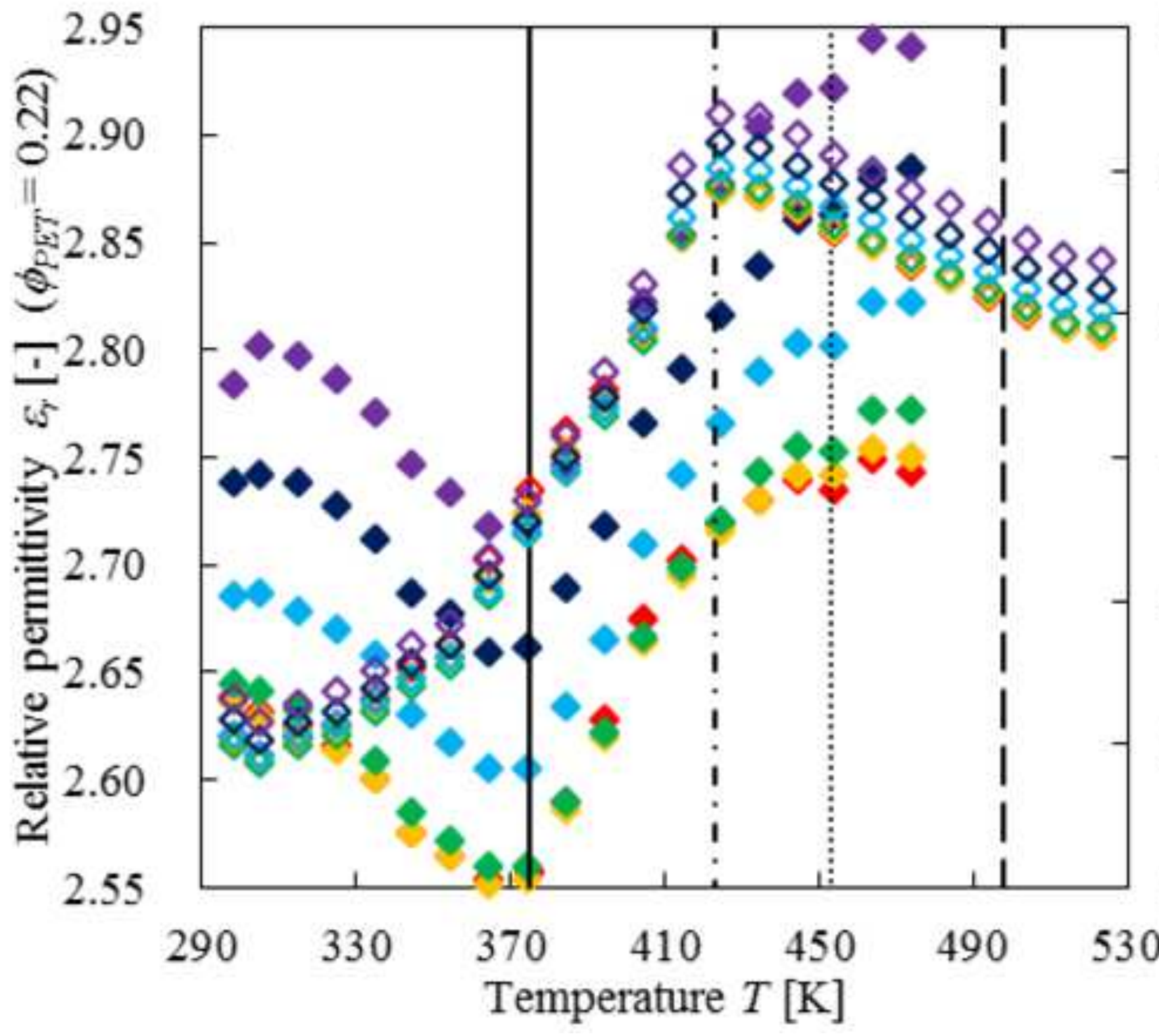

6.0

$f$

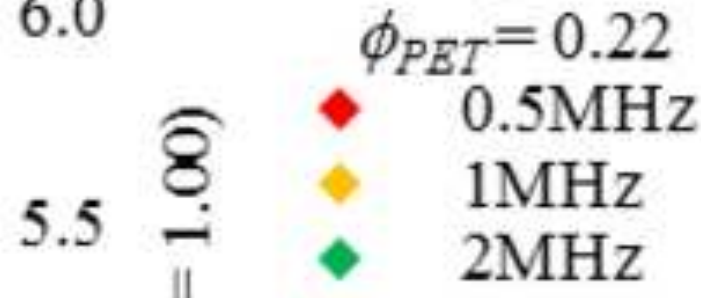

$\because 3 \mathrm{MHz}$

$5.0 \stackrel{9}{\mathrm{M} H z}$ 4.5 它 $\quad \phi_{P E T}=1.00$

4.5

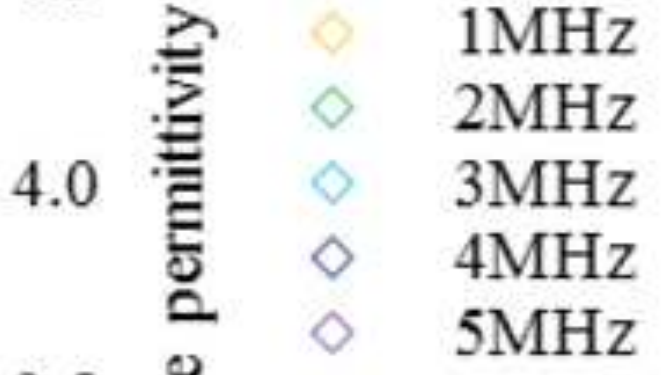

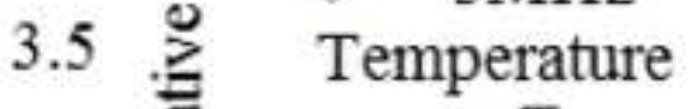

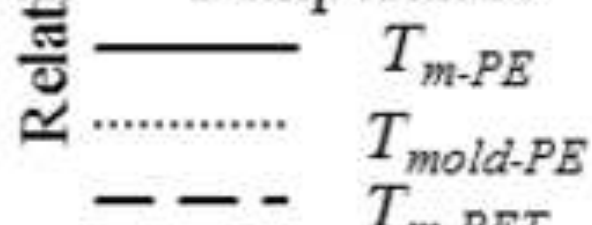

3.0

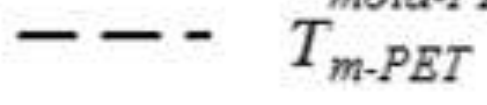

-...- Peak 


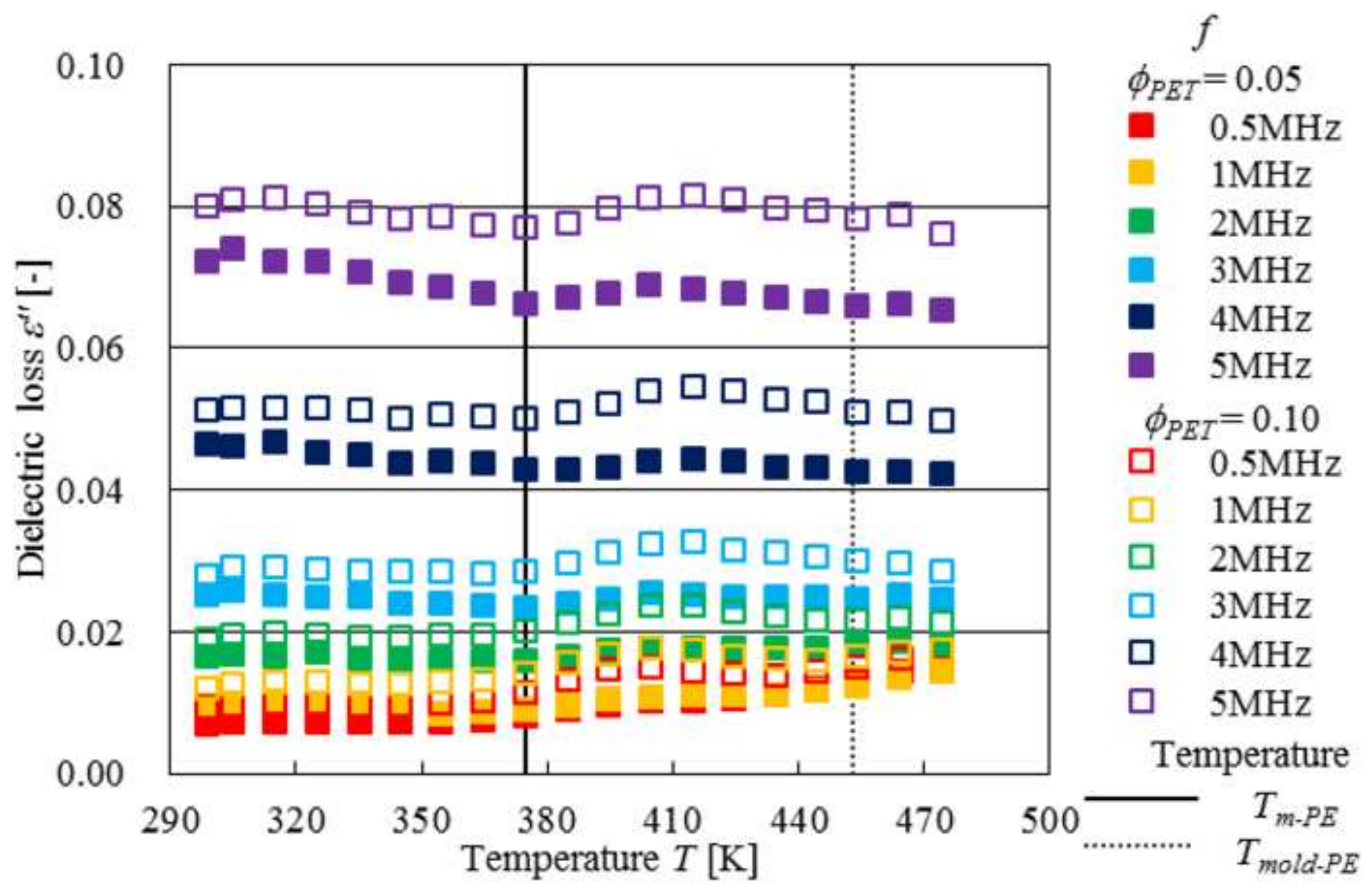




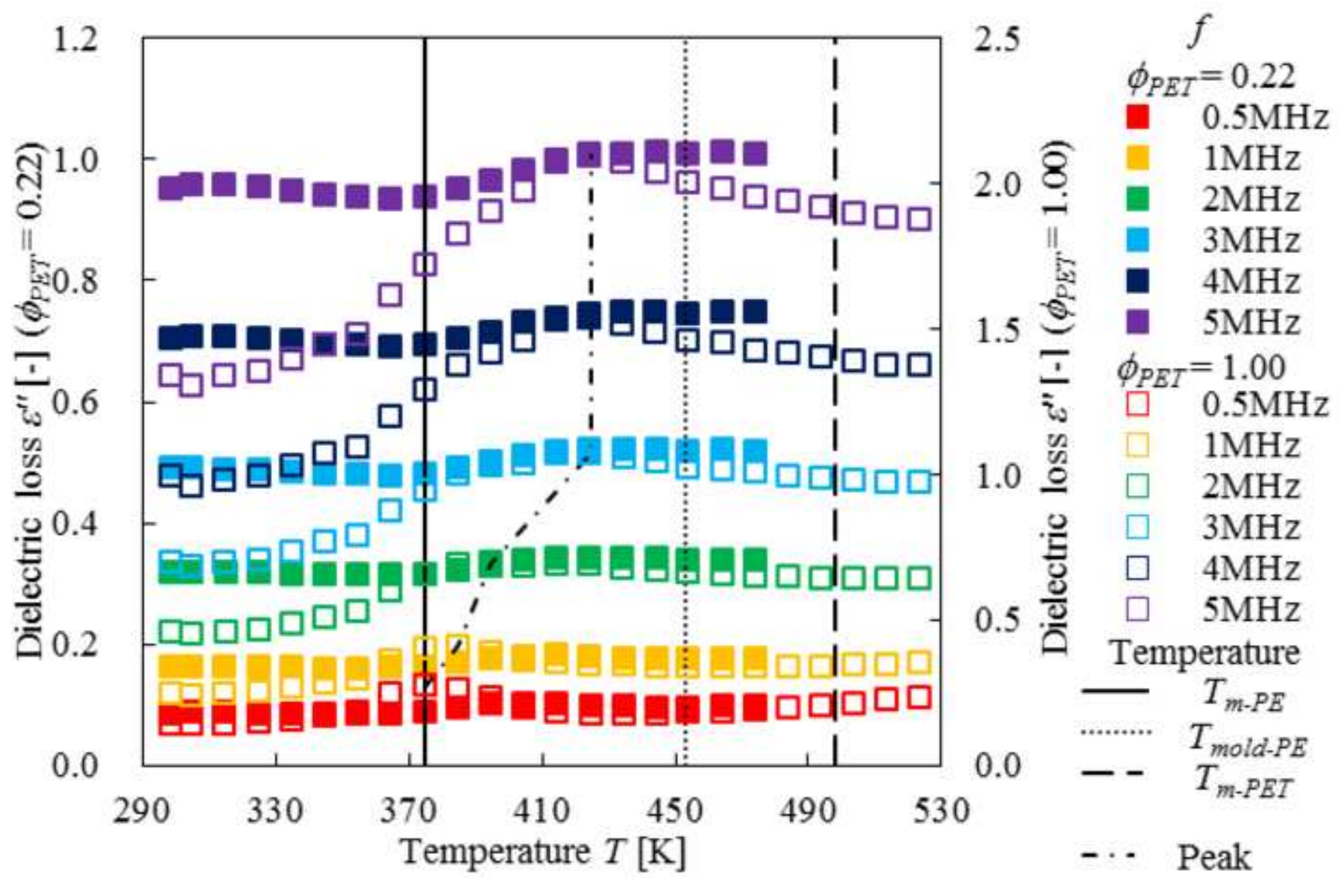




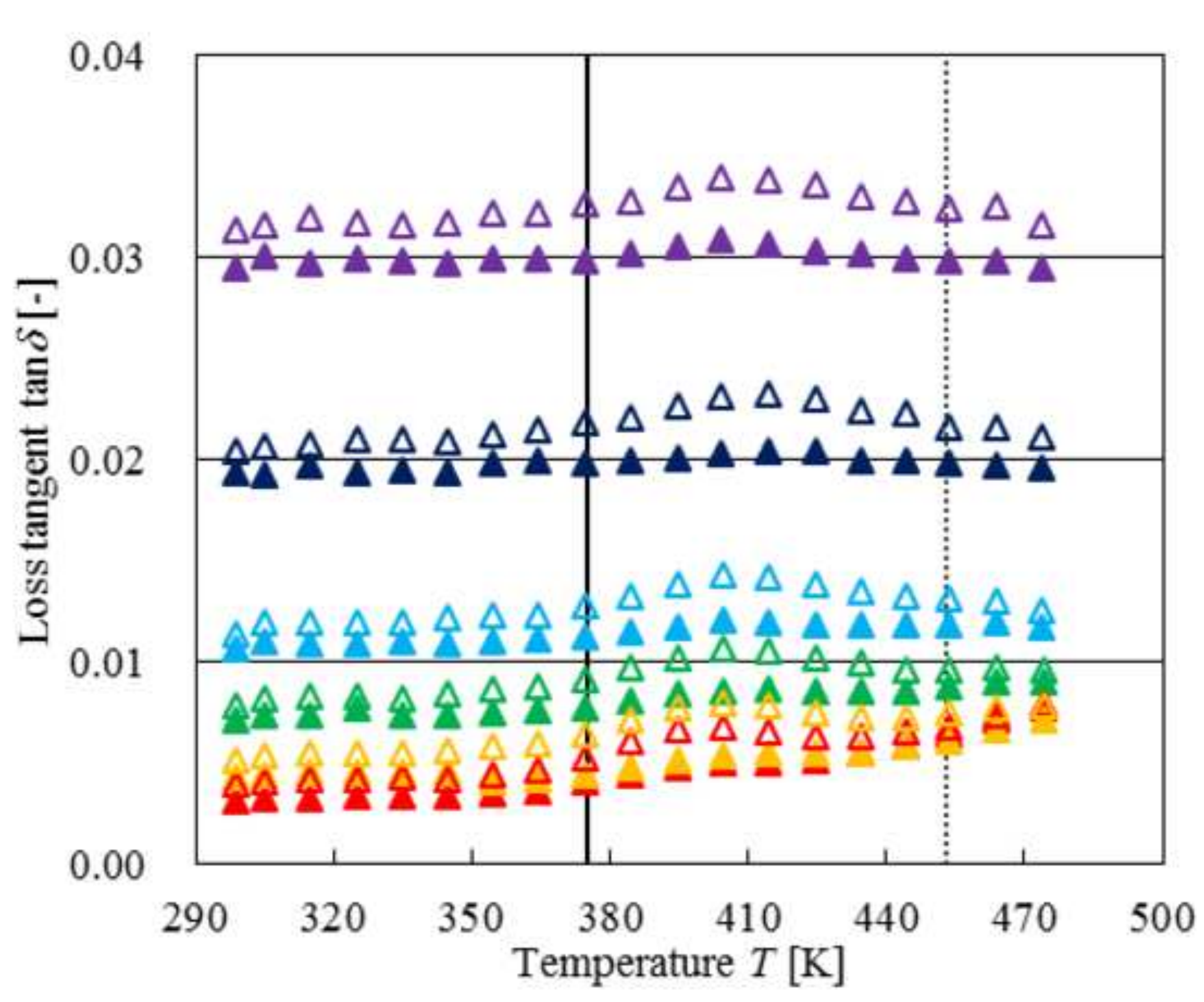

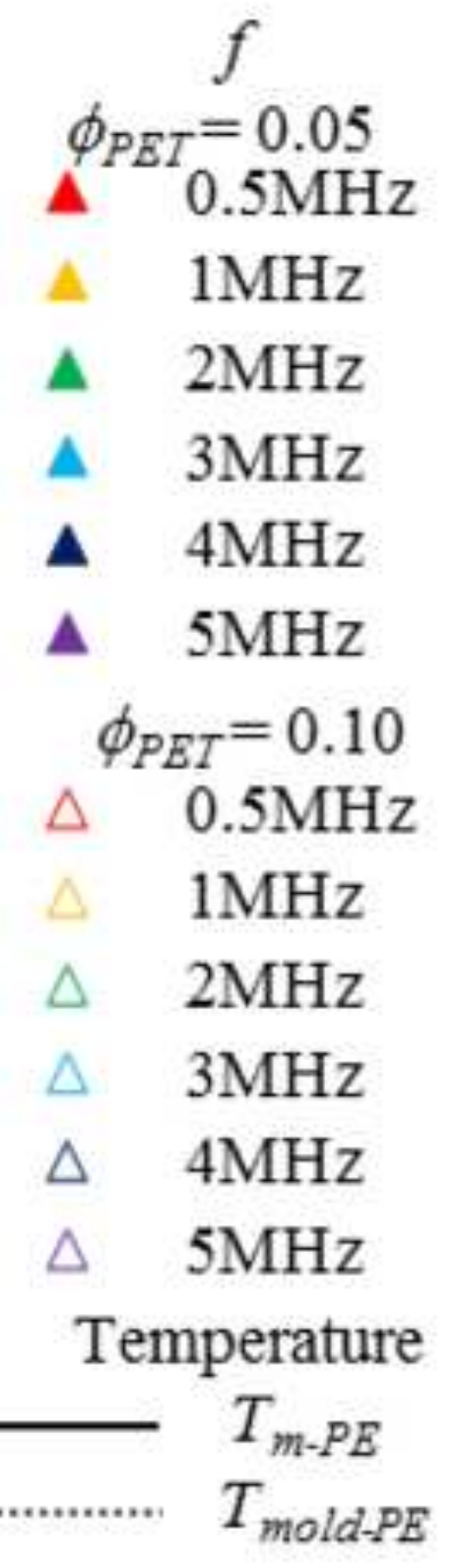




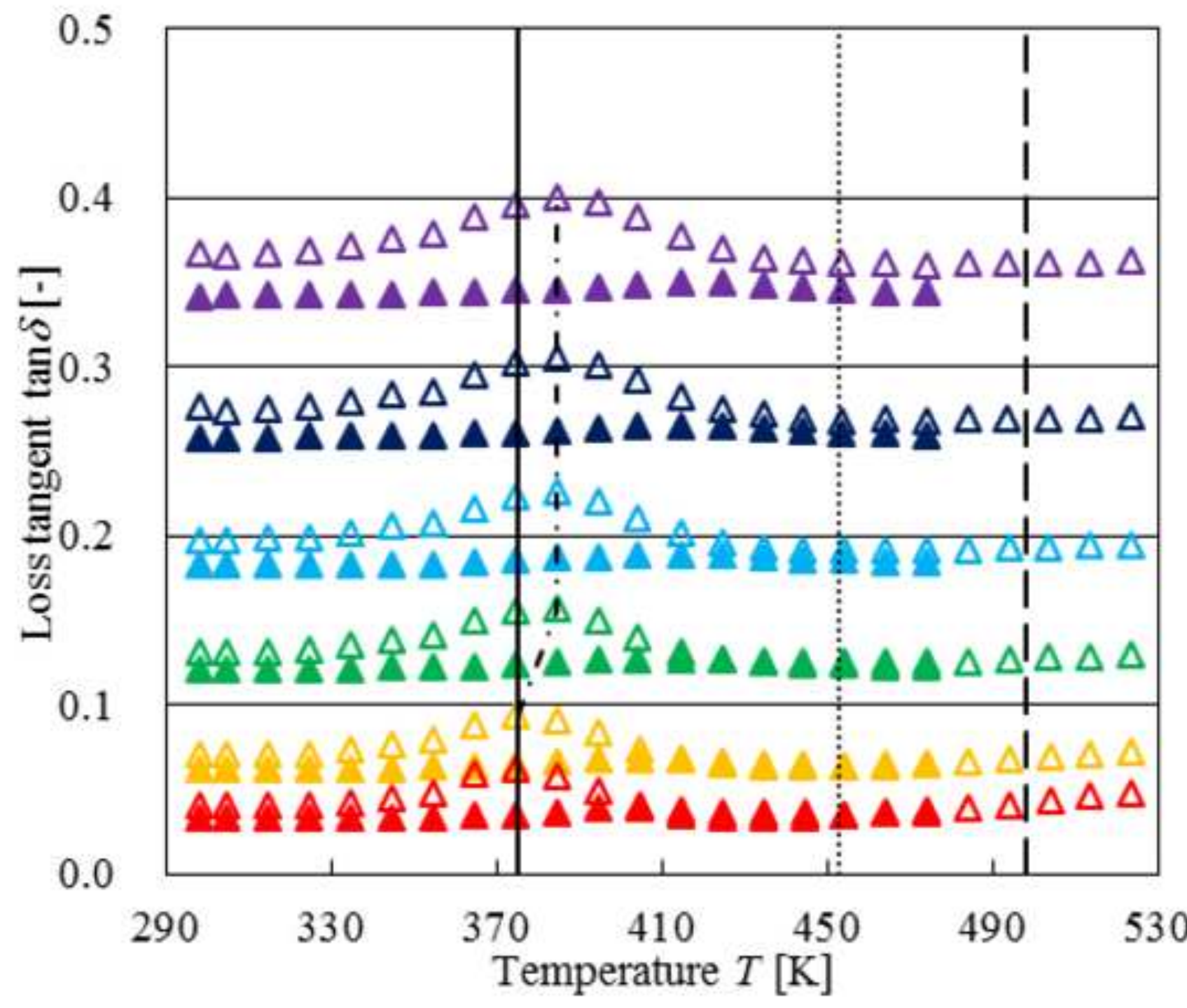

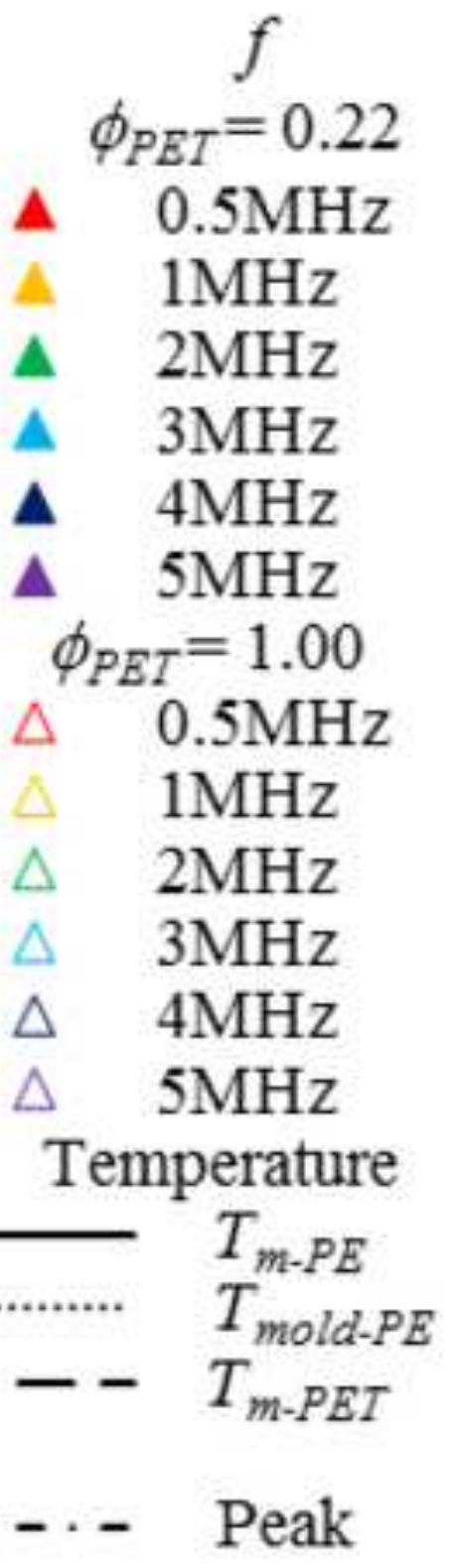




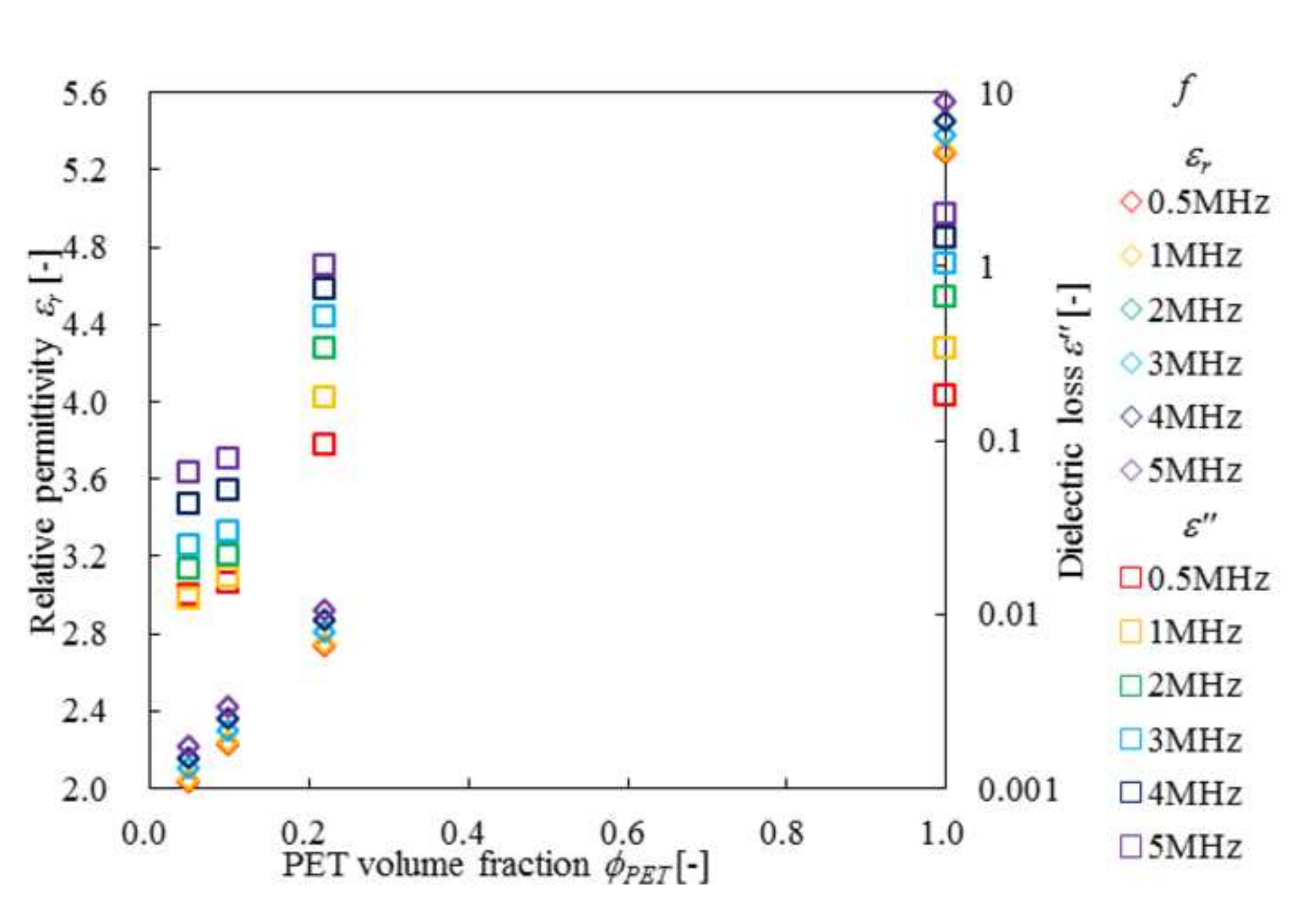

(1)




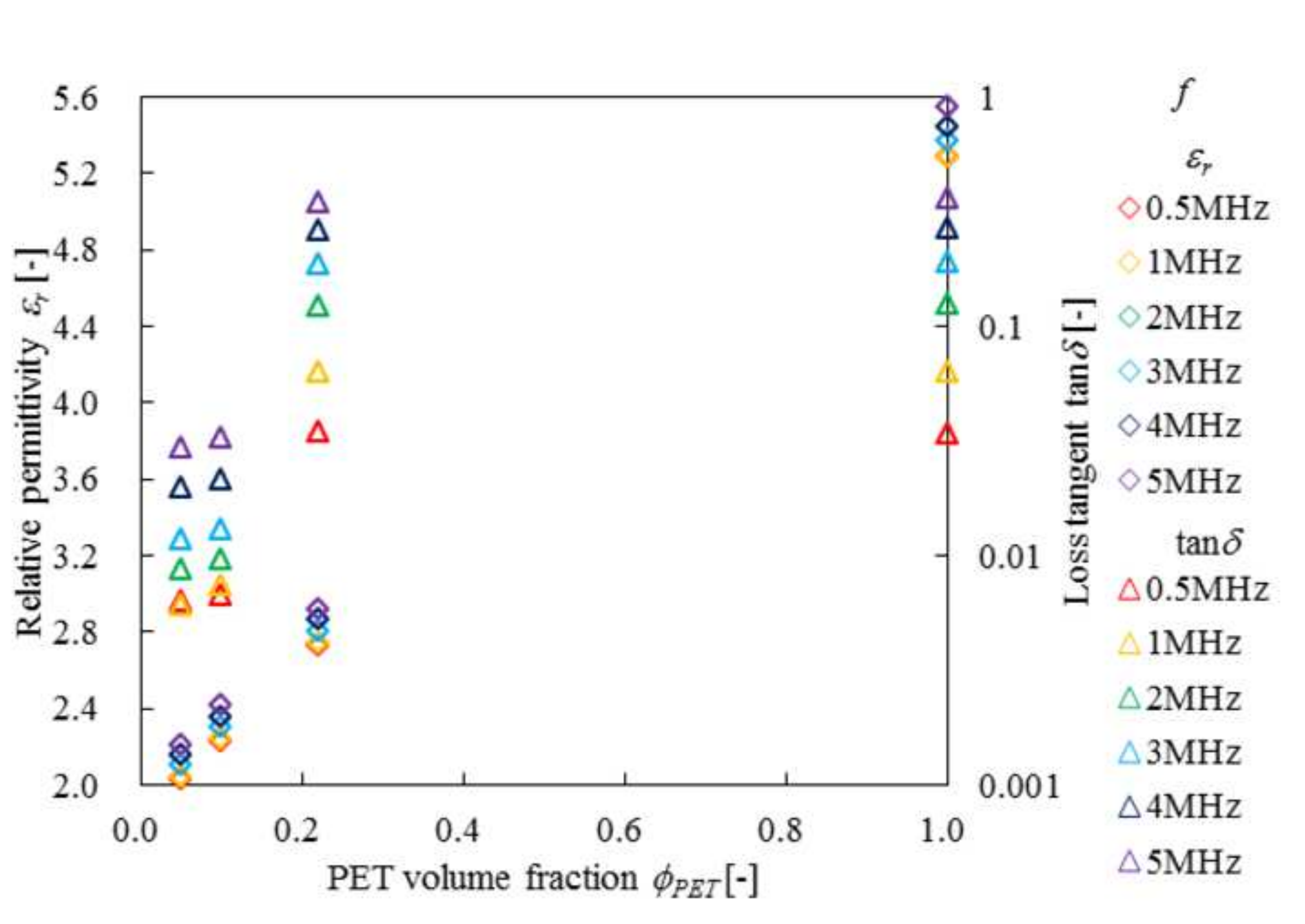

\author{
PET volume fraction $\phi_{P B r}[-]$
}

.

(⿸丆口

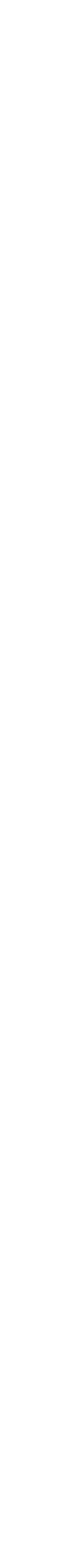

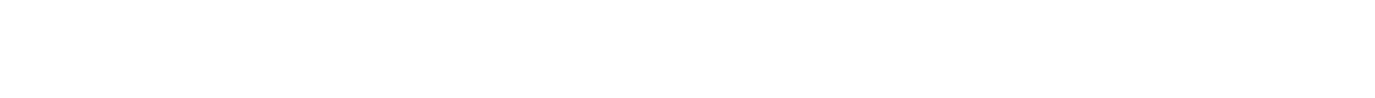




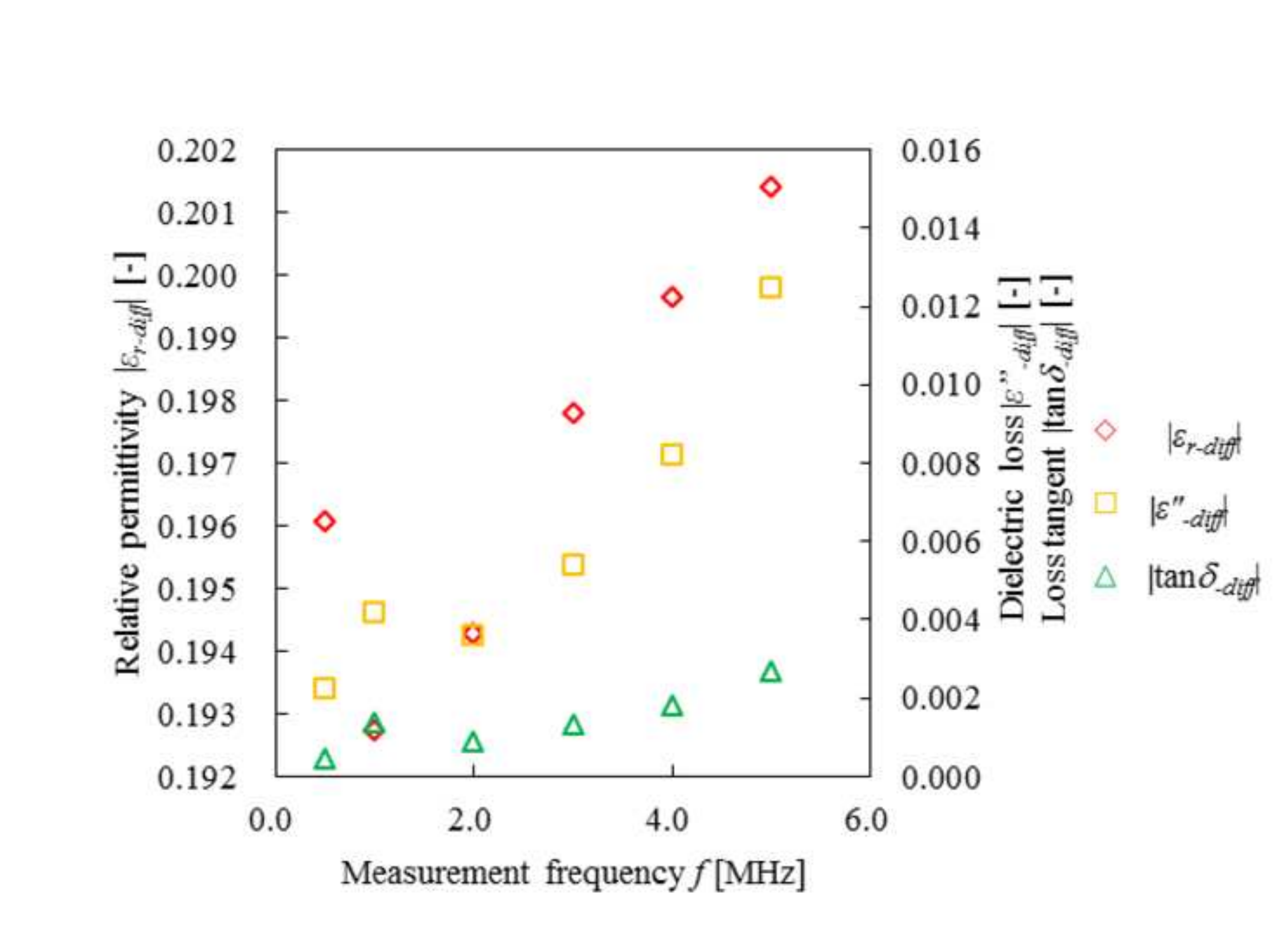




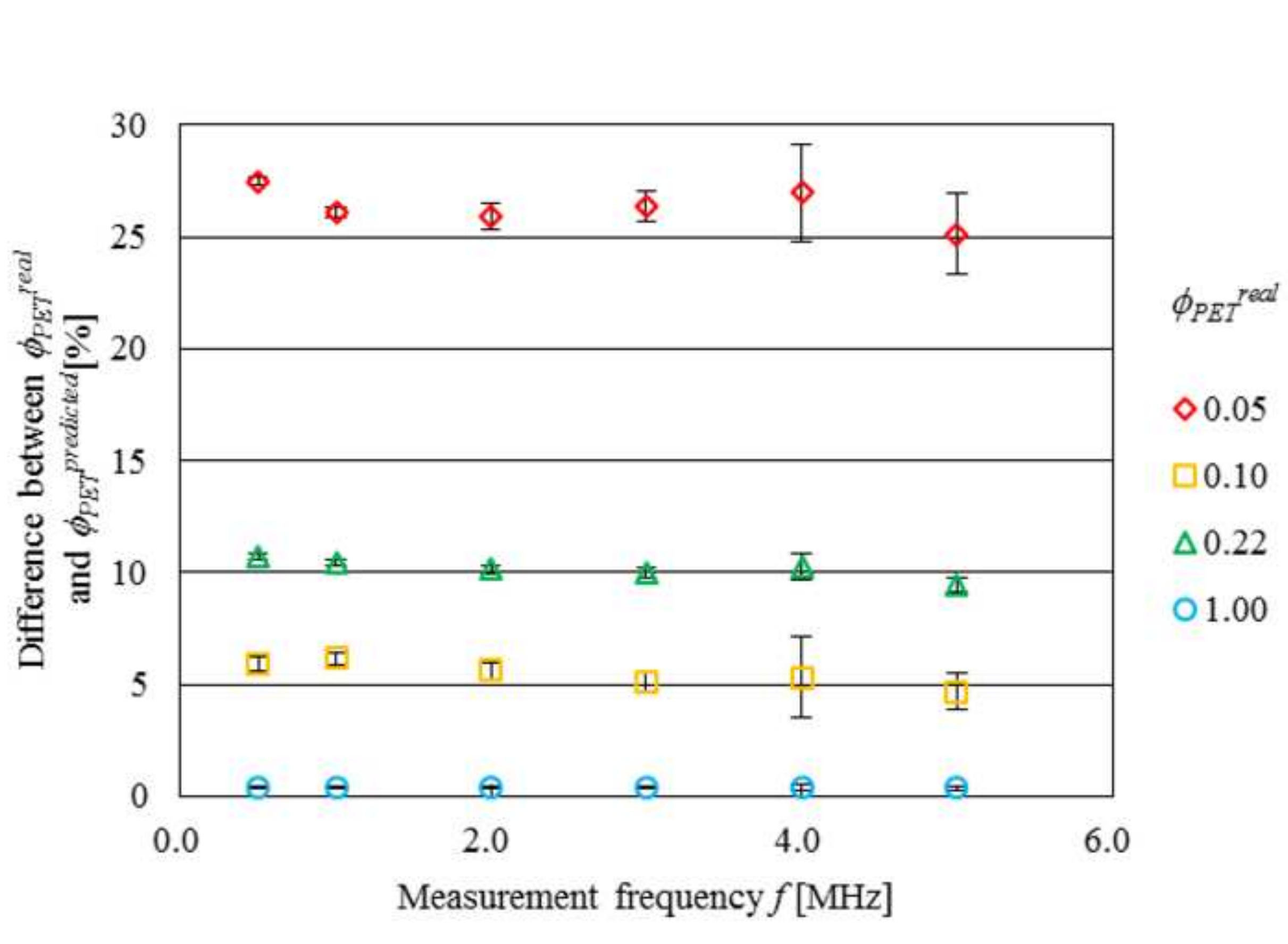

.




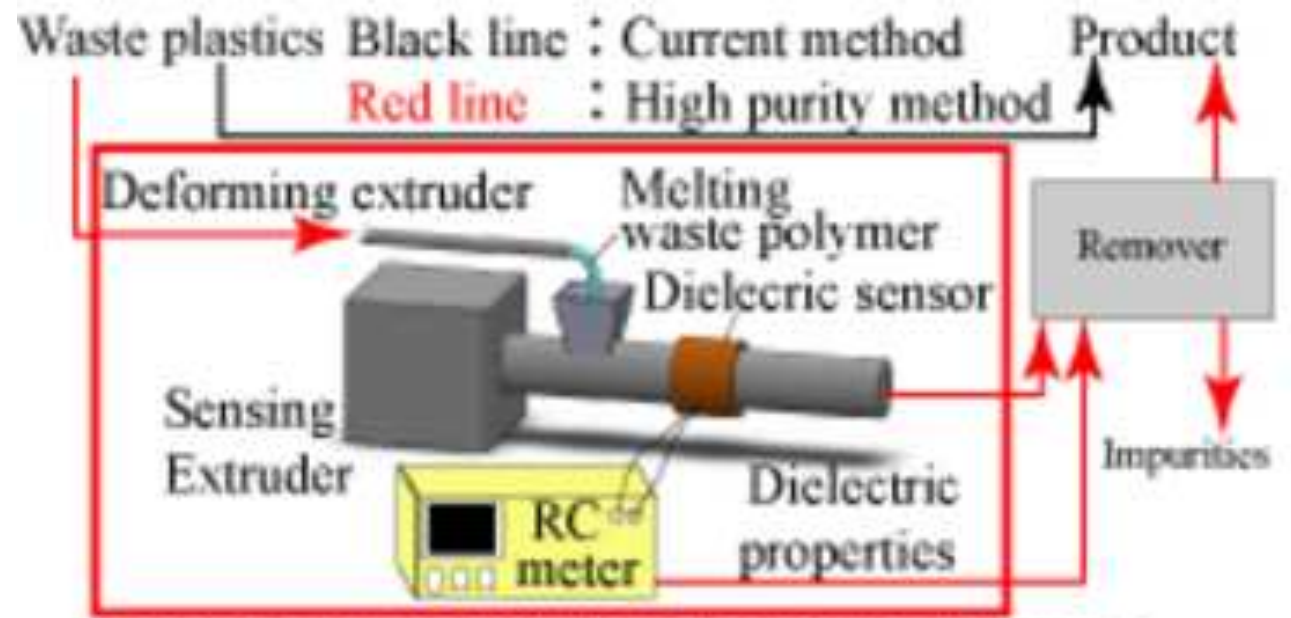

Proposed sensing technique for high performance waste plastic separation by using dielectric sensor

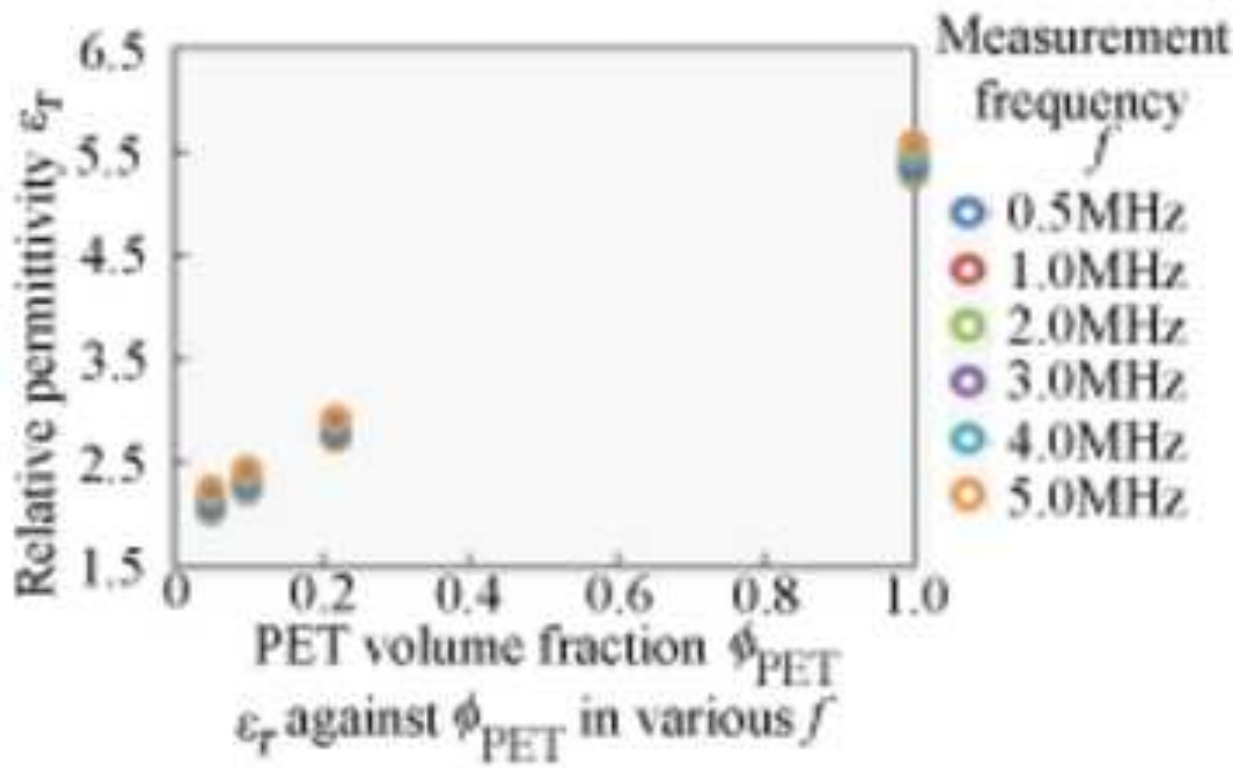

*The highest sensitivity and lincarity are $\varepsilon_{\gamma}$ and $/-5 \mathrm{MH} z$ in PE mold temperature.

"The performance to distinguish 0.05 PET volume fraction to 0.95 volume fraction of $\mathrm{PE}$ is achived in the mixed melting polymer pellets. 